\title{
Equalização Cega com Realimentação de Decisões Baseada em Redes Imunológicas Artificiais
}

\author{
Diogo C. Soriano*, Everton Z. Nadalin", Cristina Wada", Rafael Ferrarił, Ricardo Suyama, Romis R. \\ de F. Attux*
}

\begin{abstract}
Resumo- Este trabalho propõe um estratégia cega para adaptação dos parâmetros de um equalizador com realimentação de decisões (DFE). Esta abordagem, baseada no critério do modulo constante (CM) e num sistema imunológico artificial, foi testada em canais de comunicação representativos sob o ponto de vista prático. Em todos os casos, foi possível encontrar a solução ótima do critério $\mathrm{CM}$ com um número de iterações considerado bastante razoável, tendo em mente, entre outras coisas, a complexidade da ferramenta de busca.
\end{abstract}

Palavras-chave- Equalização cega, equalização com realimentação de decisões, sistemas imunológicos artificiais, computação evolutiva.

Abstract - This work proposes a blind strategy for adapting the parameters of a decision-feedback equalizer (DFE). The approach, which is based on the constant modulus (CM) criterion and on an artificial immune system, was tested under scenarios characterized by channels representative of a number of aspects relevant from a practical standpoint. In all cases, it was possible to find the global optimum of the $\mathrm{CM}$ criterion with a number of iterations considered by us to be quite reasonable in view of, inter alia, the complexity of the employed search tool.

Index Terms- Blind equalization, decision-feedback equalization, artificial immune systems, evolutionary computation.

\section{INTRODUÇÃO}

$\mathrm{U}$ MA questão de grande importância para diversos sistemas de comunicação digital é a escolha de uma metodologia de projeto de um equalizador - um filtro que busca estimar de modo tão preciso quanto possível uma mensagem transmitida sujeita a interferência intersimbólica (IIS). Tipicamente, uma metodologia dessa natureza envolve duas etapas fundamentais: a escolha da estrutura de filtragem e a determinação de um esquema de aprendizado dos coeficientes.

\footnotetext{
* Departamento de Eng. de Computação e Automação Industrial, $\dagger$ Departamento de Microondas e Óptica, Faculdade de Engenharia Elétrica e de Computação, Universidade Estadual de Campinas, São Paulo, Brasil. Emails: \{soriano, nadalin, criswada, attux\}@dca.fee.unicamp.br; \{rferrari, rsuyama\}@dmo.fee.unicamp.br; Os autores agradecem à CAPES e à FAPESP pelo apoio financeiro recebido.
}

Se tivermos em mente a idéia de estabelecer uma solução significativamente geral, surge como opção promissora a possibilidade de empregar uma combinação entre um equalizador com realimentação de decisões (DFE, do inglês decision-feedback equalizer) - uma estrutura com enorme capacidade de correção de canais lineares - e o critério do módulo constante (CM, do inglês constant modulus), uma abordagem não-supervisionada (ou seja, que não requer esquemas que provejam acesso periódico a um sinal de referência) de marcada solidez teórica [1].

O problema de otimização que decorre do emprego do critério CM no processo de escolha dos parâmeros do DFE é, não obstante, nada trivial: aspectos como a existência de mínimos locais e a ameaça da propagação de erros podem comprometer decisivamente o processo de adaptação caso se empreguem métodos clássicos como aqueles baseados no gradiente estocástico. O trabalho de Casas [2,3], inclusive, mostra que certos "maus canais" (bad channels) levam à convergência para mínimos locais caso se adote a inicialização padrão na origem.

Neste trabalho, é proposta uma abordagem para adaptação dos parâmetros do DFE via critério CM que se baseia numa ferramenta evolutiva com significativo potencial de busca local e global: um sistema imunológico artificial inspirado na proposta de de Castro e Von Zuben [4]. Para avaliar o potencial da proposta, são adotados cenários de teste compostos por diferentes canais, incluindo um bad channel de Casas [2,3], um canal de fase mista e um canal com zeros sobre o círculo unitário que, como mostraram Montalvão et al. [5], impõe sérios problemas a qualquer estrutura de filtragem que não disponha de laços de realimentação.

Este trabalho está estruturado da seguinte forma: na seção 2, é feito um breve estudo do problema de equalização baseada em realimentação de decisões; na seção 3 , é exposta a ferramenta de otimização adotada; na seção 4, discute-se a aplicação dessa ferramenta ao problema de equalização enfocado; na seção 5, discutem-se os resultados obtidos enquanto, na seção 6 , são apresentadas as conclusões e 
perspectivas.

\section{EQUALIZAÇÃO CEGA USANDO EQUALIZADORES COM REALIMENTAÇÃO DE DECISÕES}

O principal objetivo de um sistema de comunicação é permitir a troca de informação de forma adequada entre transmissor e receptor conectados a um canal que é capaz de distorcer uma mensagem enviada e corromper a informação nela contida de várias formas. $\mathrm{O}$ canal de comunicação pode, em diversos cenários práticos, ser modelado, na ausência de ruído aditivo, como um filtro linear, de tal forma que sua relação entrada-saída pode ser expressa como:

$$
x(n)=h(n) * s(n)
$$

onde $s(n)$ e $x(n)$ são, respectivamente, os sinais transmitido e recebido, $h(n)$ é a resposta impulsiva do canal, e * denota a convolução.

Uma possível abordagem para minimizar os efeitos indesejáveis que o canal pode exercer sobre a informação é o emprego de um filtro denominado equalizador. Este dispositivo é projetado com o fim de recuperar o sinal transmitido $s(n)$ a partir de sua versão possivelmente corrompida $x(n)$. Há vários dispositivos que podem fazer o papel de equalizador, desde estruturas clássicas como filtros lineares transversais até redes neurais. Dentre as alternativas existentes, um candidato de grande relevância é o equalizador com realimentação de decisões (DFE, do inglês decisionfeedback equalizer), cuja estrutura é mostrada na Figura 1, [6].

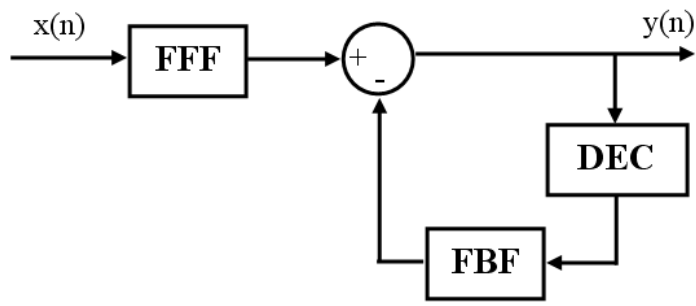

Fig.1. Estrutura do equalizador com decisão realimentada. FFF representa o filtro de alimentação direta; FBF é o filtro da malha de realimentação; DEC é o decisor; $x(n)$ é o sinal recebido; $y(n)$ o sinal de saída.

DFEs são filtros não-lineares recorrentes comumente utilizados para reduzir IIS em sistemas de comunicação. Este tipo de equalizador é basicamente composto de duas partes: um filtro de alimentação direta (FFF, do inglês feedforward filter) e um filtro de realimentação (FBF, do inglês feedback filter). O primeiro opera diretamente sobre o sinal recebido, enquanto o segundo processa amostras provenientes de um dispositivo de decisão, o que possibilita escrever a saída do equalizador como sendo:

$$
y(n)=\mathbf{f}^{\mathrm{T}} \cdot \mathbf{x}(n)-\mathbf{b}^{\mathrm{T}} \cdot \mathbf{v}(n)
$$

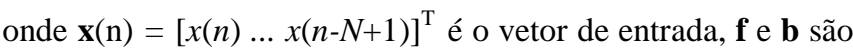
os vetores de parâmetros dos filtros de alimentação direta e de realimentação, com tamanhos $N$ e $M$, respectivamente, e $\mathbf{v}(n)$ $=\operatorname{dec}\left\{[y(n-1) \ldots y(n-\mathrm{M})]^{T}\right\}$, com a função $\operatorname{dec}($.$) indicando o$ mapeamento realizado pelo decisor.

A existência do filtro de realimentação pode melhorar de forma substancial a capacidade de compensação do canal pelo DFE em comparação com o clássico equalizador de resposta ao impulso finita (FIR). Além disso, a natureza não-linear do DFE permite, em teoria, a perfeita inversão de canais que não sejam de fase mínima, o que não seria possível mesmo se um filtro linear com resposta ao impulso infinita (IIR) fosse utilizado.

Embora a utilização do DFE ofereça uma série de vantagens, a determinação dos parâmetros dos filtros (vetores $\mathbf{f}$ e b) pode ser, efetivamente, problemática. Existem duas grandes abordagens possíveis para a adaptação dos parâmetros: uma supervisionada, baseada no conhecimento das amostras de $s(n)$, e outra não-supervisionada, baseada apenas em aspectos estatísticos da informação que se deseja recuperar.

Tendo em vista a idéia de atuar num cenário tão geral quanto possível, técnicas supervisionadas não foram aqui utilizadas, uma vez que as mesmas necessitam de uma sequiência de treinamento nem sempre disponível. A opção, neste espírito, foi considerar a adaptação dos coeficientes de um DFE através do critério do módulo constante, que é, possivelmente, a abordagem não-supervisionada mais estudada na última década [1].

A combinação entre uma estrutura não-linear e um critério não-supervisionado leva a complexas superfícies de custo, superfícies que podem conter mínimos locais associados a taxas de erro de bit significativas mesmo na ausência de ruído, o que justifica, em casos práticos em que há uma demanda por níveis elevados de desempenho, a utilização de um procedimento de adaptação alternativo.

O procedimento adotado neste trabalho para realizar o processo de busca de modo a contornar as dificuldades supramencionadas é uma técnica evolutiva que pode ser vista como uma versão (voltada à codificação real dos indivíduos) do algoritmo de otimização baseado no princípio da seleção clonal proposto por de Castro e Von Zuben [4]. A escolha dessa técnica se justifica por seu interessante equilíbrio entre capacidade de busca global e parcimônia, bem como pelos resultados interessantes obtidos previamente com ferramentas imunológicas no contexto de processamento de sinais [7]. Para esclarecer estes pontos, passemos a uma breve análise do algoritmo.

\section{SisteMAS IMUNOLÓGICOS ARTIFICIAIS}

Os sistemas imunológicos artificiais (SIAs) pertencem à classe das técnicas de computação inspiradas em conceitos biológicos (mais especificamente, em conceitos do sistema imunológico) que vêm sendo aplicadas com sucesso na solução de problemas de engenharia [8].

Em especial, neste trabalho, utiliza-se uma técnica de busca construída sobre idéias como seleção clonal e maturação de 
afinidade [9], técnica esta que pode levar a um bom desempenho no que se refere à taxa de convergência global em problemas marcados por uma significativa multimodalidade.

Os conceitos de seleção clonal e maturação de afinidade estabelecem que, quando um organismo é invadido por um antígeno (e.g. vírus ou bactéria), células específicas do sistema imune reconhecem o elemento exógeno e são selecionadas para se proliferarem (dando origem a um processo de clonagem) com taxas proporcionais à afinidade (grau de compatibilidade ou reconhecimento) desses marcadores pelos antígenos. Segue-se a este processo a reprodução da população, na qual a geração produzida será sujeita a mutações com taxas inversamente proporcionais à afinidade dos marcadores pelos antígenos. Essa geração mutada pode eventualmente apresentar indivíduos com maior afinidade, o que tende a lhes conferir maior tempo de vida em relação às demais células na evolução da população.

Para converter essas idéias num algoritmo de otimização efetivo, é preciso fazer algumas considerações. Primeiramente, cada possível solução de um problema corresponde a um indivíduo, um vetor com valores reais que representa, em termos simples, a estrutura de uma célula imune. A qualidade de um indivíduo (fitness), dada pela função custo que se deseja otimizar, vincula-se à idéia de afinidade anticorpo-antígeno $[4,10]$. Por fim, há também no algoritmo um passo de inserção periódica de novos indivíduos aleatoriamente gerados que pode ser relacionado ao conceito de edição de receptores [10]. O pseudocódigo para o laço principal do procedimento de adaptação proposto é apresentado Figura 2.

\section{Enquanto critério de parada não é atendido}

1) Determine a afinidade de todos indivíduos da população.

2) Gere $N c$ clones (cópias) de cada indivíduo da população.

3) Estabeleça a mutação para cada indivíduo da população, bem como para seus clones, de forma inversamente proporcional à afinidade.

4) Selecione o melhor indivíduo de cada grupo formado pelo indivíduo original e por seus clones (geração mutada de cada "pai").

5) Insira periodicamente (a cada $N p$ gerações) $\mathrm{Ni}$ indivíduos gerados aleatoriamente na população no lugar dos piores indivíduos.

\section{Fim enquanto}

Fig. 2. Pseudocódigo para o algoritmo imunológico utilizado.

A população inicialmente utilizada é composta de um conjunto de vetores reais gerados aleatoriamente. O critério de parada pode ser um valor previamente determinado para a função custo, ou mesmo um número fixo de gerações / iterações. A mutação é dependente da afinidade, e é estabelecida como:

$$
c^{\prime}=c+\alpha \cdot N(0,1)
$$

$$
\alpha=\left(\frac{1}{\beta}\right) \cdot \exp \left(-J_{F I T}\right)
$$

onde c' é uma versão mutada de c, $N(0,1)$ é uma variável aleatória Gaussiana de média zero e variância unitária, $\beta$ é um parâmetro que controla o decaimento da função exponencial inversa que regula a mutação, e $J_{F I T}$ é a afinidade do indivíduo normalizada no intervalo [0,1].

O estágio de seleção clonal, descrito nos passos 2, 3 e 4 do pseudocódigo acima, pode ser interpretado como uma busca adaptativa local, enquanto a inserção de novos indivíduos gerados aleatoriamente, descrita no passo 5, aumenta significativamente o poder de busca global da ferramenta, o que pode ser decisivo quando se lida com problemas multimodais. Esta estratégia de inserção contribui para a rápida convergência para a solução ótima, embora essa abordagem deva ser vista com cautela, uma vez que a retirada dos piores indivíduos pode, eventualmente, gerar certo "elitismo" na população.

Deve-se enfatizar que o algoritmo é, tendo em mente a classe de ferramentas evolutivas, relativamente simples do ponto de vista operacional - sendo dotado de uma complexidade comparável à de um algoritmo genético clássico - o que foi uma preocupação fundamental à luz das aplicações vislumbradas neste trabalho. Elementos de uma análise mais detalhada do potencial de busca do algoritmo podem ser encontrados em [4].

\section{APLICAÇÃO DO SisteMA IMUNOLÓGICO ARTIFICIAL AO PROBLEMA DE EQUALIZAÇÃO COM REALIMENTAÇÃO DE DECISÕES}

Com o intuito de avaliar a performance do algoritmo imunológico proposto na adaptação de DFEs, uma série de experimentos representativos de diferentes aspectos foram realizados. Assumiu-se aqui que o sinal transmitido pertence ao alfabeto binário $( \pm 1)$ com amostras independentes e identicamente distribuídas. O filtro FFF foi considerado como sendo um simples ganho unitário (um procedimento indicado e justificado por Casas em [2,3] à luz de uma análise que envolve a estrutura dos canais de comunicação) e os coeficientes b do filtro FBF passaram a ser os parâmetros com respeito aos quais se buscou minimizar a função custo dada pelo critério CM:

$$
J_{C M}=E\left[\left(1-y^{2}(n)\right)^{2}\right]
$$

que é estimada por meio de médias temporais usando janelas de amostras de $y(n)$.

Como o algoritmo proposto é uma ferramenta de otimização que busca por máximos de uma dada função, é necessário transformar os mínimos da função custo $J_{C M}$ em máximos, o que foi feito por meio da expressão:

$$
J_{F I T}=\frac{1}{1+J_{C M}}
$$

O algoritmo adotou como critério de parada a existência de um indivíduo com afinidade $\left(J_{F I T}\right)$ superior a 0.999 , uma vez 
que o os coeficientes ótimos são encontrados para $J_{F I T}$ igual a 1. Para todas as simulações realizadas, o tamanho da população foi fixado em 5 indivíduos, o valor de $\beta$ foi atualizado a cada iteração como sendo $100 \cdot J_{F I T}, N i$ foi fixado em 2 e $N p$ em 20, com a inserção de 2 novos indivíduos gerados aleatoriamente no lugar dos 2 indivíduos de menor afinidade.

\section{RESUltados}

No primeiro conjunto de simulações foi considerado um canal de fase mínima com função de transferência dada por $h_{1}(z)=1+0.9 z^{-1-} 0.8 z^{-2}$ e solução ótima $\mathbf{b}=[0.9-0.8]^{\mathrm{T}}$. Esse canal é um dos bad channels estudados por Casas, os quais levam à convergência para mínimos locais caso se inicialize um método de gradiente na origem. Trata-se, portanto, de um canal para cuja equalização a abordagem proposta é particularmente relevante.

No segundo conjunto de simulações, foi considerado um canal com resposta ao impulso $h_{2}(z)=1+0.1 z^{-1}-1.8 z^{-2}-0.9 z^{-3}$ cuja solução ótima é dada por $\mathbf{b}=\left[\begin{array}{lll}0.1 & -1.8 & -0.9\end{array}\right]^{\mathrm{T}}$. Este canal foi escolhido exatamente por ser de fase não-mínima, uma vez que tais canais são particularmente relevantes no âmbito do teste de algoritmos cegos por não se prestarem diretamente à equalização via filtragem de erro de predição. O caráter de fase não-mínima também é muito interessante para teste de um DFE, pois uma vantagem dessa estrutura sobre um filtro IIR linear é exatamente a sua capacidade de inverter canais que têm zeros fora do círculo unitário. Por fim, o terceiro conjunto de simulações considera um canal com zeros em 1 e $-1, h_{3}(z)=$ $1-1 z^{-2}$, com solução ótima dada por $\mathbf{b}=\left[\begin{array}{ll}0 & -1\end{array}\right]^{\mathrm{T}}$. Esse canal pertence à classe de canais discutidos em Montalvão et al. [5]. Tais canais se caracterizam por darem origem a problemas de equalização equivalentes a tarefas de classificação impossíveis para estruturas sem realimentação, devido à existência de estados coincidentes. Por esse motivo, tais modelos são muito interessantes quando se contempla o uso de DFEs.

A tabela I mostra os parâmetros utilizados em cada conjunto de simulações (coeficientes do canal e número de clones), bem como os valores médio, mínimo e máximo obtidos para os coeficientes do filtro FBF ao longo de 100 realizações para cada um dos canais. Observa-se que para todos os canais analisados, os valores médios, mínimos e máximos foram muito próximos à solução ótima para o respectivo caso, o que indica que o algoritmo teve um desempenho bastante adequado em todas as realizações e para todos os canais. Notase ainda que a redução do número de clones utilizados na busca não afetou de forma substancial a solução final encontrada pelo método, o que é bastante animador, uma vez que significa que a técnica é capaz de operar satisfatoriamente do ponto de vista de desempenho sem um grande dispêndio de recursos computacionais.

Na Tabela II, evidencia-se a eficiência do método de busca em termos de velocidade de convergência, uma vez que é mostrado o número de gerações (iterações) até a convergência da média, mínimo e máximo para o conjunto de 100 realizações para cada canal. Nota-se que, em todos os casos, o número de iterações requerido para a convergência foi satisfatório (em comparação com índices associados a métodos de busca via gradiente, por exemplo), embora a redução do número de clones por indivíduo tenha tido o efeito de reduzir em cerca de $50 \%$ a velocidade de convergência. Esse efeito é esperado se levarmos em conta que a redução do número de clones diminui a capacidade de busca local associada a cada geração.

TABELA I

PARÂMETRos de SimUlaC̃̃̃o E COEFICIENTES Do FILTRo FBF ObTIDOS

\begin{tabular}{|c|c|c|c|c|c|}
\hline Canal & Clones & Coefic. & Média & Mínimo & Máximo \\
\hline \multirow{4}{*}{$h_{1}(z)$} & \multirow{2}{*}{5} & $b_{0}$ & 0,898 & 0,885 & 0,916 \\
\hline & & $b_{1}$ & $-0,801$ & $-0,815$ & $-0,785$ \\
\hline & \multirow{2}{*}{2} & $b_{0}$ & 0,900 & 0,884 & 0,916 \\
\hline & & $b_{1}$ & $-0,802$ & $-0,815$ & $-0,784$ \\
\hline \multirow{6}{*}{$h_{2}(z)$} & \multirow{3}{*}{5} & $b_{0}$ & 0,102 & 0,085 & 0,115 \\
\hline & & $b_{1}$ & $-1,794$ & $-1,813$ & $-1,785$ \\
\hline & & $b_{2}$ & $-0,898$ & 0,914 & $-0,885$ \\
\hline & \multirow{3}{*}{2} & $b_{0}$ & 0,103 & 0,085 & 0,115 \\
\hline & & $b_{1}$ & $-1,797$ & $-1,812$ & $-1,785$ \\
\hline & & $b_{2}$ & $-0,899$ & $-0,914$ & $-0,885$ \\
\hline \multirow{4}{*}{$h_{3}(z)$} & \multirow{2}{*}{5} & $b_{0}$ & 0,000 & $-0,016$ & 0,016 \\
\hline & & $b_{1}$ & $-1,000$ & $-1,015$ & $-0,985$ \\
\hline & \multirow{2}{*}{2} & $b_{0}$ & 0,000 & $-0,015$ & 0,016 \\
\hline & & $b_{1}$ & $-1,001$ & $-1,016$ & $-0,984$ \\
\hline
\end{tabular}

TABELA II

ParÂMETROS DE SimUlaÇ̃̃o E TAXAS DE CONVERGÊNCIA DO SISTEMA IMUNOLÓGICO ARTIFICIAL

\begin{tabular}{c|c|c|c}
\hline \multirow{2}{*}{ Canal } & Clones & $\begin{array}{c}\text { Média de } \\
\text { iterações }\end{array}$ & $\begin{array}{c}\text { Máximo de } \\
\text { iterações }\end{array}$ \\
\hline \multirow{2}{*}{$h_{1}(z)$} & 5 & 82 & 148 \\
\cline { 2 - 4 } & 2 & 206 & 333 \\
\hline \multirow{2}{*}{$h_{2}(z)$} & 5 & 112 & 242 \\
\cline { 2 - 4 } & 2 & 273 & 497 \\
\cline { 2 - 4 }$h_{3}(z)$ & 5 & 80 & 114 \\
\cline { 2 - 4 } & 2 & 200 & 322 \\
\hline
\end{tabular}

A figura 3 mostra uma realização típica da adaptação dos coeficientes do filtro FBF para o canal $h_{1}(z)$, evidenciando a evolução temporal da afinidade $\left(J_{F I T}\right)$ associada ao melhor indivíduo e da afinidade média da população. Observa-se que o melhor indivíduo produz uma rápida convergência para a vizinhança do valor ótimo de custo, enquanto a afinidade média da população apresenta flutuações a cada 20 iterações, o que decorre da inserção de dois novos indivíduos gerados aleatoriamente na população no lugar dos dois indivíduos de menor afinidade. A distância relativamente grande entre o melhor indivíduo e a média da população é um indicador de uma boa exploração do espaço de busca, uma vez que ilustra a diversidade da afinidade dos indivíduos.

A figura 4 mostra as curvas de nível para a função custo $J_{F I T}$ associada ao canal $h_{1}(z)$ bem como a trajetória do melhor indivíduo até sua convergência para o máximo local. Observase que a inicialização do indivíduo na bacia de atração de um mínimo local não prejudica a qualidade da solução obtida, em contraste com o que tenderia a ocorrer caso uma técnica baseada nas derivadas da função custo fosse utilizada. 


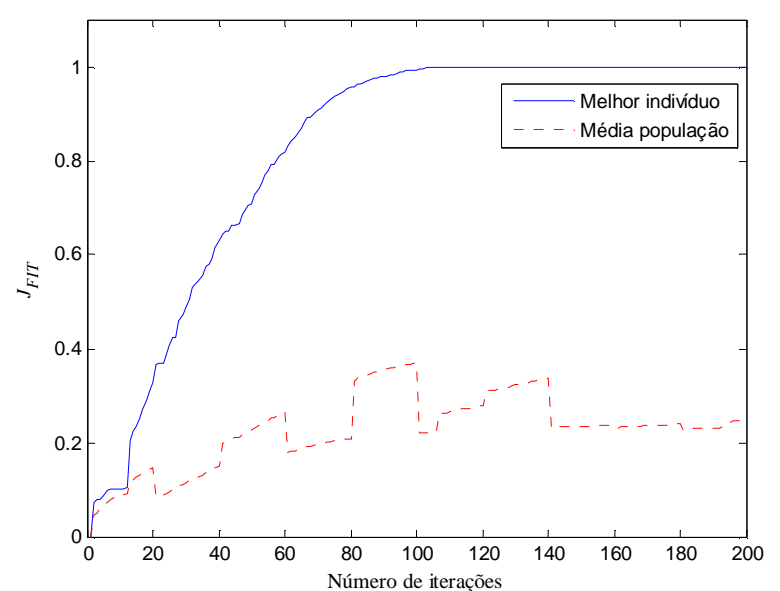

Fig. 3. Evolução das afinidades calculadas pelo método de busca para o melhor indivíduo da população e da afinidade média da população para cada iteração do algoritmo para o canal $h_{1}(z)$.

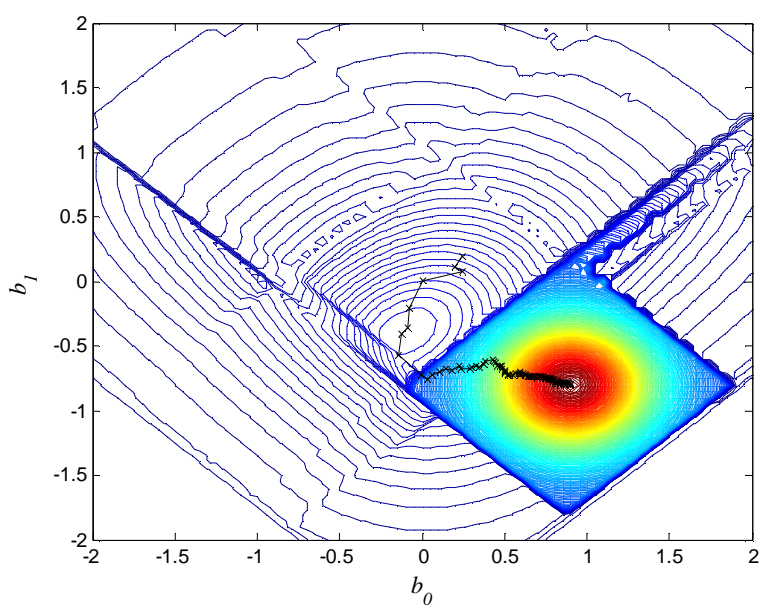

Fig. 4. Curvas de nível da função custo $J_{F I T}$ associada ao canal $h_{1}(z)$. Os pontos em preto mostram a trajetória do melhor indivíduo até a solução ótima.

De modo análogo ao realizado para o canal $h_{1}(z)$, a figura 5 mostra a evolução das afinidades para o melhor indivíduo e para a média da população para o canal $h_{3}(z)$. A análise dessas curvas leva às mesmas conclusões expostas junto à figura 3 . A figura 6 mostra as curvas de nível para este canal, bem como a trajetória do melhor indivíduo para a solução ótima. Mais uma vez, observa-se que o método de busca permite o escape de uma bacia de atração inferior (embora nela pareça não haver um mínimo), o que ilustra mais uma vez a eficiência da abordagem.

\section{CONCLUSÕES}

Foi apresentada neste trabalho uma abordagem para adaptação dos coeficientes de um equalizador com decisão realimentada que une o critério do módulo constante a um algoritmo imunológico capaz de realizar eficientemente buscas em contextos multimodais.

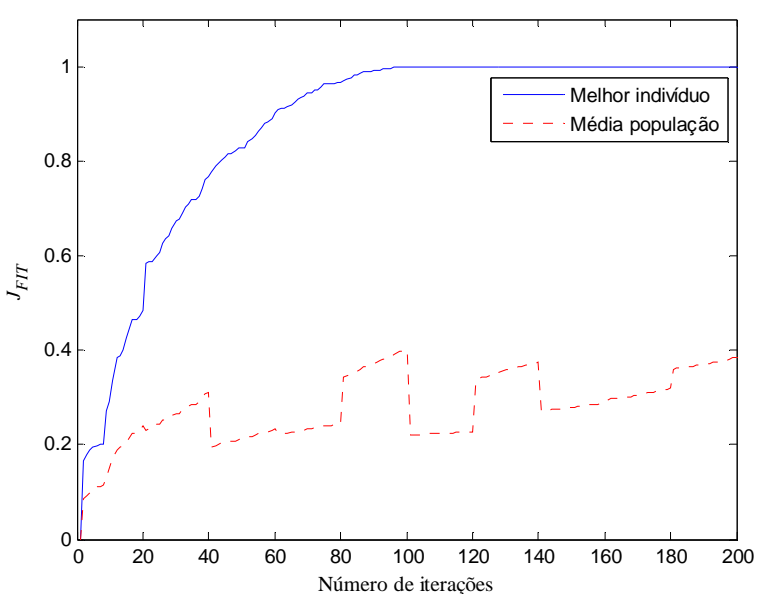

Fig. 5. Evolução das afinidades calculadas pelo método de busca para o melhor indivíduo da população e da afinidade média da população para cada iteração do algoritmo para o canal $h_{3}(z)$.

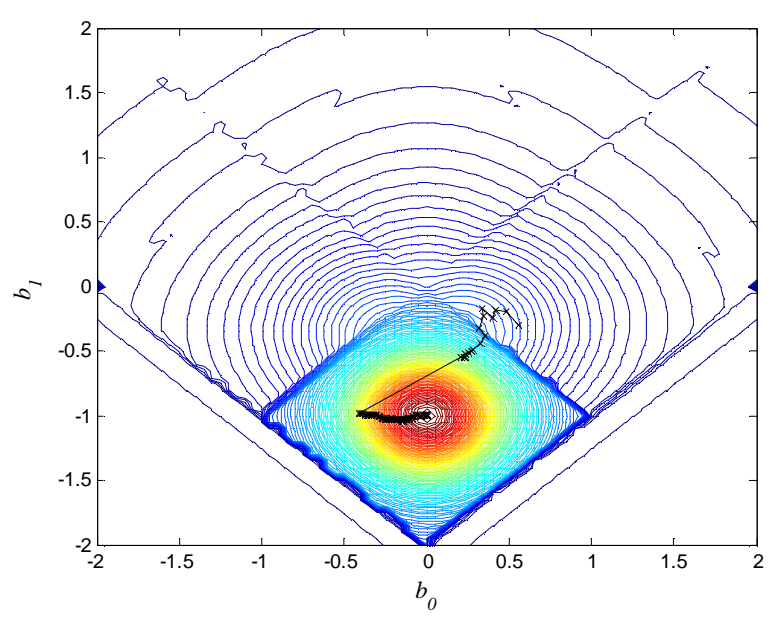

Fig. 6. Curvas de nível da função custo $J_{F I T}$ associada ao canal $h_{3}(z)$. Os pontos em preto mostram a trajetória do melhor indivíduo até a solução ótima.

Para todos os canais explorados, chegou-se à convergência para a solução ótima em $100 \%$ das simulações realizadas e numa quantidade de iterações compatível com aquele associado a técnicas clássicas de gradiente, o que indica que a metodologia é bastante promissora em termos de desempenho e, dentro dos canais aqui estudados, é capaz de contornar os problemas de convergência para mínimos locais abordados por Casas [2,3]. Por outro lado, a técnica é, sem dúvida, mais custosa que um algoritmo de gradiente estocástico tanto do ponto de vista do modus operandi relacionado à busca quanto em termos do processo requerido de estimação da função custo. Essas considerações indicam dois caminhos importantes para pesquisas futuras: 1) investigar técnicas ainda mais simples em termos de mecanismos de busca e estimação de custo e 2) estender essa idéia a problemas similares em áreas como desconvolução e separação de fontes, que, em muitos casos, não impõem restrições significativas de operação em tempo real. 


\section{REFERÊNCIAS}

[1] C.R. Johnson, P. Schniter, T.J. Endres, J.D. Behm, D.R. Brown, R.A. Casas, "Blind Equalization Using the Constant Modulus Criterion: A Review", Proceeding of the IEEE, vol. 86, no. 10, pp. 1927-1950, 1998.

[2] R.A. Casas, "Blind Adaptative Decision Feedback Equalization", MS Thesis, Cornell University, 1997.

[3] R.A. Casas, Z. Ding, R.A. Kennedy, C.R. Johnson, R. Malamut, "Blind Adaptation of Decision Feedback Equalizers Based on the Constant Modulus Algorithm", Conference Record of the Twenty-Ninth Asilomar Conference on Signals, Systems and Computers, Pacific Grove, 1995.

[4] L.N. de Castro and F.J. Von Zuben, "Learning and Optimization Using the Clonal Selection Principle", IEEE Transactions on Evolutionary Computation, vol. 6, no.3, pp. 239-251, 2002.

[5] J. Montalvão, B. Dorizzi, J.C.M. Mota, "Some Theoretical Limits of Efficiency of Linear and Nonlinear Equalizers", Revista da Sociedade Brasileira de Telecomunicações, vol. 14, no. 2, pp. 85-92, 1999.

[6] J.G. Proakis, "Digital Communications", $4^{\text {th }}$ ed., New York: McGrawHill, 2001

[7] R.R. Attux, M.B. Loiola, R. Suyama, L.N. de Castro, F. J. Von Zuben, J.M.T. Romano, Blind Search for optimal Wiener Solutions Using an Artificial Immune Network Model, EURASIP Journal of Applied Signal Processing: Special Issue on Genetic and Evolutionary Computation for Signal Processing and Image Analysis, no. 8, pp. 740-747, July, 2003.

[8] L. N. de Castro and J. I. Timmis, Artificial Immune Systems: A New Computational Intelligence Approach, Springer-Verlag, 2002.

[9] G. L. Ada and G. J. V. Nossal, "The Clonal Selection Theory", Scientific American, 257(2), pp. 50-57, 1987.

[10] L.N. de Castro and J.I. Timmis, "An immune network model to multimodal function optmization", in Proc. Of the Int. Conf. On Evolutionary Computation, Workshop on Artificial Immune Systems, vol. 1, pp. 699-674, 2002. 